\title{
Research on Dimensional Measurement Based on Sub-pixel Edge Detection
}

\author{
Yang Weidong ${ }^{1, a}$, Wang Jiaxing ${ }^{2, b}$, Peng Kai ${ }^{1, c}$ and Sun Dan ${ }^{1, d}$ \\ ${ }^{1}$ School of Mechanical Engineering, Hebei University of Technology, Tianjin, China \\ ${ }^{2}$ Shenyang Machine Tool Co. Ltd., Shenyang, China \\ aywd_stephen@163.com, bjiaxing_wang@smtcl.com, ckaip_tju@163.com, \\ dsundan063089@126.com
}

Keywords: Sub-pixel; Edge Detection; Gaussian Fitting; Moment

\begin{abstract}
With the development of modern industry, image measurement technology with its high speed ,high precision and non-contact advantages receives high-profile attention. In the machine vision system the size of the process of mechanical parts measured, it is found that the accuracy of the edge position directly influence the accuracy of the measurement results. According to the research on the current sub-pixels positioning of image processing technology, this paper firstly makes a theory analysis and research about sub-pixel location methods based on gray level moment theory and the theory of Gaussian fitting. Then through the parts size measurement experiment, under the premise of contrast location with classical edge detection operators, some groups of data are extracted respectively compared to both of the detection performance and accuracy.Thus it provided the reference for the sub-pixels edge detection algorithm in the actual application.
\end{abstract}

\section{Introduction}

There are many advantages like non-contact, high efficiency, low cost and so on by using visual techniques for dimensional measurements of parts. The principle is based on obtaining image pixel size(in pixels) by collecting the edges of images, then transforming the measurement results into an actual size through the camera calibration, so the edge detection is the key technology of measurement.The classical edge detection operators have LOG operator, canny operator, sobel operator and so on[1]. These operators are simple in form, but the positioning accuracy is just in the pixel level. With the increasing demands of accuracy, the original classic edge detection methods cannot be satisfied with the acuuracy requirement of the machine vision measurment at present, the sub-pixel edge positioning methods are paid more and more attention, and become a hot research direction in recent image processing technology. Sub-pixel edge positioning is based on the edge detection, the measurement results are further refined under the pixel accuracy,which was proposed by Hueckek [2]. The fitting methods are used to achieve the sub-pixel precision positioning, then according to the different positioning principle it is developed into interpolation, moment method, fitting, fitting of surface, wavelet transform and many other kinds of methods[3]. This paper is focused on two sub-pixel positioning methods of gray moment and Gaussian fitting, and the comparison of the pros and cons of the both methods by the experiment of measuring the size of workpiece are detailed.

\section{Principle of Sub-pixel Edge Location Based on Moment Theory in Gray}

The moment method for edge detection in gray image was first proposed by the Alij. Tabatabai and O.Robert Mitchell in 1984[4]. They said that for one-dimensional edge patterns an ideal edge is fit to the data by matching moment, that is to say,the moment of the actual edge and the ideal edge in image are equal,that is invariant moment.In the moment theory,a scan line across the step edge is characterized by a monotone increasing sequence, an ideal edge is a sequence of the brightness value 
$h_{1}$ in one side and $h_{2}$ in another hand,shown as Fig.1.Fig.1(a) is the input data and Fig1. (b)is sample data and ideal edge matched with the first three order gray moments.

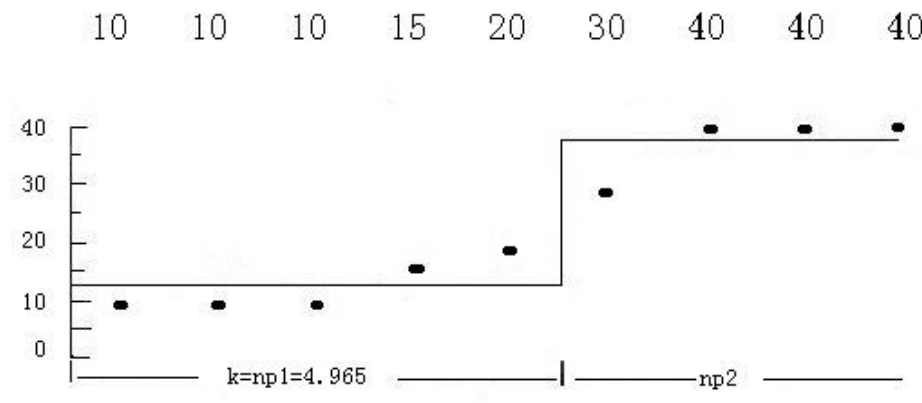

Fig.1 model of the ideal edge and actual edge

According to the definition of moment in gray image, the first three order gray moments of the input data $X_{j}(j=1,2,3 \cdots n)$ are defined as :

$$
\bar{m}_{j}=\frac{1}{n} \sum_{j=1}^{n} X_{j}^{i} \quad i=1,2,3
$$

If we let $\mathrm{k}$ denote the coordinate of the ideal edge, the first three order moments between the input data and the output data are given below :

$\sum_{j=1}^{2} p_{j} h_{j}^{i}=\bar{m}_{i}$

Where $p_{1}=\frac{k}{n}, \sum_{j=1}^{2} p_{j}=1$

Because the moment of the actual edge and the ideal edge in image are equal, Eq. 3 can be determined by solving Eq.1and Eq.2simultaneously.

$$
\left\{\begin{array}{l}
p_{1} h_{1}+p_{2} h_{2}=\frac{1}{n} \sum_{i=1}^{n} x_{i} \\
p_{1} h_{1}^{2}+p_{2} h_{2}^{2}=\frac{1}{n} \sum_{i=1}^{n} x_{i}^{2} \\
p_{1} h_{1}^{3}+p_{2} h_{2}^{3}=\frac{1}{n} \sum_{i=1}^{n} x_{i}^{3}
\end{array}\right.
$$

Thus,

$$
k=n\left[1-\frac{1}{2}\left(1+\bar{s} \sqrt{\frac{1}{4+\bar{s}^{2}}}\right)\right]
$$

Where $\bar{s}=-\frac{\bar{m}_{3}+2 \bar{m}_{1}^{3}-3 m_{1} m_{2}}{\bar{\sigma}^{3}}, \bar{\sigma}^{2}=\bar{m}_{2}-\bar{m}_{1}^{2}$

From Eq. 4 we see that a sub-pixel measurement locating the ideal position of edge can be obtained according to the pixel gray value and the corresponding position.

\section{Principle of Sub-pixel Edge Location Based on Gaussian Fitting Theory}

The principle of determining the sub-pixel edge by using Gaussian fitting theory [5]is: in the gradient direction of image edge the gray gradient is distributed by Gaussian curve, the extreme point of Gaussian curve is the edge position. In the digital image, all data are discrete, the point coordinates of grayscale having the biggest changes are not often the whole pixel. In order to find the point which the grayscale having the greatest changes between the pixels ,the Gaussian fitting is an effective way. Gaussian curve is given by: 


$$
y=\frac{1}{\sqrt{2 \pi}} e^{-\frac{(x-\mu)^{2}}{2 \sigma^{2}}}
$$

Eq.6 is derived by doing logarithmic transformation for Eq.5:

$$
\ln y=-\frac{(x-\mu)^{2}}{2 \sigma^{2}}+\ln \frac{1}{\sqrt{2 \pi \sigma}}
$$

Eq.5 is a form of $y=a x^{2}+b x+c$ curve, fitting out a,b,c through the least square principle, then $\mu=-\frac{b}{2 a}$ is obtained which is the sub-pixel edge position. This approach has a rotation invariance, so there are no specific requirements for selecting the line direction of gray value. The core idea of Gaussian fitting is similar to the curve fitting[6], but they use different fitting model.

\section{Experiments}

Data Preprocessing and Coarse Location in Image.The rectangle part width of $39.00 \mathrm{~mm}$ is used for the experiments. During the process of measuring the actual image, because of the interference coming from the optical devices and external condition for capturing images, the preprocess are used for the image to eliminate the interference of the gray value which is invalid, before the sub-pixel analysis for the image.Then classical edge detection operator for the coarse positioning of the image edge are used to determine the sampling interval of gray values of the sub-pixel positioning. Fig.2(a) is the actual collection images used the image acquisition system, Fig.2(b) is the results of pixel-level edge detection by using the sobel operator. From the graph it can be seen that sobel operator is well used for coarse positioned on the edge.
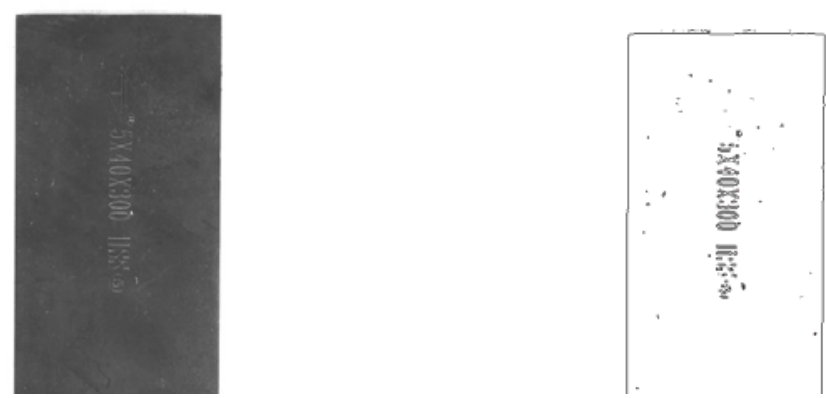

Fig.2 results of coarse location by sobel

Subpixel Location Analysis. For example, each group sample 11 points. The coarse location to ensure left edge gray value for the image sampling area are from 228 to 238 column, in the same way right edge from 440 to 450 column. Need to notice, Gray moment theory model is the step function of rising edge. Through the data analysis and experiment, it can be found that the rise and decline of the edge of the step function use different $\bar{s}$ in the process of using gray moment for the sub-pixel.

Coordinate the range is $[1, \mathrm{n}]$ ( $\mathrm{N}$ is the number of data collection) when using gray moment positioning edge.Finally the edge of the position should be tranformed between the image coordinates and the corresponding world coordinates based on on the location of sampling points in the image coordinate,thus the coordinates of actual sample points are obtained.

For example, the left edge of the first data is the image of the 228 listed data, so it can be calculated the left edge $y$-coordinate is 234.82888 ,in the same way the right edge $y$-coordinate is 445.1341 .The distance between the edge is the width dimension of the parts, the measurement units of data analysis are pixels.

In the process of the Gaussian fitting method for sub-pixel positioning the adjacent pixel gray value need to do subtraction and take the natural logarithm for fitting. Therefore theoretical analysis of the model requires that the adjacent pixel gray values are different, so as to achieve the requirements of the non-zero difference between adjacent pixels gray value. In the process of actual 
sampling, the gray value changing trends and ideal model is different, it will be the situation of the adjacent pixels gray value equal. Therefore, when using the Gaussian fitting for the edge detection, the first step need to filter the data collected, and then do Gaussian fitting processing. The result is the left edge of 234.4230, the right edge of 444.8536 .

The above calculated result units are pixels. In order to make the final measurement results and actual size units consistent, our system calibration experiment is accomplished. The standard of calibration method is used in the test. Measured width of $15 \mathrm{~mm}$ pixel size target subject is 81 , you can get a length and pixel transformation factor $\mathrm{k}=0.1852 \mathrm{~mm} /$ pixel, taking these measurements and the size of the transformation, these measurement results are shown in Table 1.

Table1 the results of moments theory, Gaussian fitting theory and sobel theory

\begin{tabular}{l|llll}
\hline Method & $\begin{array}{l}\text { Coordinates } \\
\text { left edge }\end{array}$ & $\begin{array}{l}\text { of } \\
\text { right edge }\end{array}$ & $\begin{array}{l}\text { Dcoordinates } \\
\text { ristance (pixel) }\end{array}$ & Dimensions (mm) \\
\hline $\begin{array}{l}\text { Moments theory } \\
\begin{array}{l}\text { Gaussian fitting } \\
\text { theory }\end{array}\end{array}$ & 234.8288 & 445.1341 & 210.3053 & 38.9485 \\
Sobel theory & 234 & 445.3536 & 210.9306 & 39.0643 \\
\hline
\end{tabular}

\section{Summary}

Moment method and fitting method for sub-pixel edge in positioning is the most commonly used method. The two kinds of typical methods are analyzed in this paper. It is proved that using sub-pixel location is feasible to measure the size by the experiments. Gray moment and Gaussian fitting method can be achieved sub-pixel positioning, then use of statistical methods can effectively improve the positioning accuracy and reduce positioning errors. However, Gaussian fitting method of data collection are more restricted, and need to do effective data filtering, so the use of gray moment method is relatively easy to locate the edge for positioning and measurement of mechanical parts.

\section{Acknowledgements}

This work was financially supported by Nature Science Research Project of Education Department of Hebei Province (Grant No. 2011215)

\section{References}

[1] Rafael C. Gonzalez, Richard E. Woods: Digital image Processing Using MATLAB (Publishing House of Electronics Industry, U.S.A. 2004).

[2] Hueckel M.F. : Journal of the ACM Vol.20( 1973),p.634

[3] Wu Jigang ,Bin Hongzan: China Mechanical EngineeringVol.8(2009),p.297.

[4] Ali J.Tabatbai,O.Robert Mitchell: IEEE Trans Pattern Anal Machine Intell Vol.PAMI-6(1984),p.188.

[5] Shang Yaceng, Chen Jing, Tian Jun-wei: Journal of Computer Applications Vol31(2011) p.179.

[6] Angela Cantatore, Alfredo Cigada, Remo Sala, et al. :Measurement Vol.42(2009), p. 1226. 